\section{Measurement of Small Quantities of Calcium by Radiometric Precipitation}

RADIOMETRIC precipitation is in principle an extremely sensitive method of analysis, but in practice the sen. sitivity is usually limited by the efficiency of separation of the precipitate and its parent solution ${ }^{1,2}$. An adapta. tion of the technique of chromatography affords a quick and simple method both of phase separation and of washing the precipitate with a small volume of liquid. I have used this method to measure concentrations of calcium as low as 10 p.p.m. in less than $1 \mathrm{mg}$ of solution.

In this method equal quantities of nitric acid containing the calcium sample and of 1 per cent fluoride solution labelled with radiofluorine are measured in a length of fine bore polythene tubing attached to a microsyringe and then mixed and temporarily stored in a small polythene bubble. The solution is then spotted on a strip of chromatographic paper where calcium fluoride is precipitated when its $p \mathrm{H}^{\text {is }}$ raised by blowing on ammonia. This method of raising the $p \mathrm{H}$ is much to be preferred to that of adding liquid as the latter tends to move the solution without mixing well with it. After precipitation some 1,000 p.p.m. fluoride solution is allowed to run along the paper until the excess activity has moved away from the precipitate, which remains stationary. A fluoride solution has to be used for the irrigation, rather than water, as about $10^{-2} \mathrm{ml}$. runs through the precipitate and this quantity of water would dissolve $10^{-7} \mathrm{~g}$ of calcium from calcium fluoride. Finally the paper is dried and the piece containing the precipitate is cut off so that the activity of the precipitate can be measured.

The quantity of calcium in an unknown sample must be determined from a calibration curve of standard calcium samples (Fig. 1) because, although the activity is directly proportional to the calcium concentration over $a_{0}$ range of several hundredfold, the measured activities of precipitates from standard calcium solutions containing 1 per cent of fluoride are roughly half of those expected on the grounds of chemical equivalence and the known specific activity of the fluoride. Some of the activity is lost by exchange of fluoride during the irrigation and some of the calcium is precipitated as calcium hydroxide when the $p \mathrm{H}$ rises abruptly. The first reaction causes only a uniform reduction in the activities of a set of precipitates, as would the second if the rate of absorption of ammonia were the same for each sample. However, random varia. tions in the quantity of fluoride precipitated with calcium

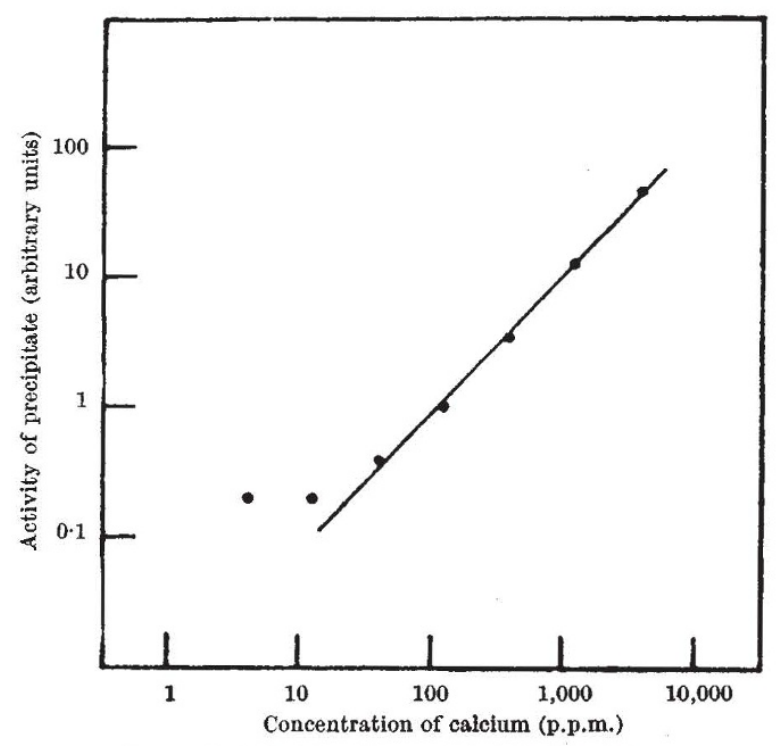

Fig. 1. Labelled fluoride precipitated with calcium are reduced to about 5 per cent if phosphoric acid, a powerful buffering agent, is present in the solution at a level of a few per cent. The precipitate with buffer is again a mixture, as the phosphates of calcium are only slightly soluble, but its composition will be determined mainly by the ratio of fluoride and phosphate concentra. tions. Further, at this level phosphoric acid has the advantage of swamping the effect of any phosphate in the unknown calcium samples.

Any substance which is present in the unknown sample and is capable of forming an insoluble compound with either calcium or fluoride might, in principle, interfere with this method of analysis. However, small quantities of anions can be masked in the way described here for phosphate: by the addition of a larger quantity of the interfering anion to all the calcium solutions so that the precipitate is a mixture of constant composition. Inter. ference by substances with fluorides of low solubility is troublesome; the effect of magnesium, for example, is complex and if it is present at a level of more than onetenth of the calcium concentration the method is unreliable.

Before use the chromatographic paper must be washed in a dilute solution of either ethylenediamine tetraacetic acid or hydrochloric acid to remove a background contamination which is probably calcium. Even so, traces of fluoride stick to the paper limiting the sensitivity of the analysis. Solutions containing less than 10 p.p.m. of calcium, the level at which the activity of the precipitate is comparable with the background activity left in the paper, must be concentrated. This can be done without risk of contamination by freezing the solution in an unsealed polythene bubble and then drying under vacuum.

The principle of this method should be widely applic. able; the main limitations being probably in handling small quantities of liquid and in finding suitable chromatographic paper.

I thank Mr. I. N. Tully for his help in the development of this technique.

Department of Physics,

University of Birmingham.

${ }^{1}$ Thackray, MI., Nature, 190, 434 (1961).

2 Scott, B. F., and Driseoll, W. J., Radioisotopes in the Physical Sciences and Industry, 339 (International A tomic Energy Agency, Vienna, 1962).

\section{Phosphorescence in Plastics}

A RECENT communication from Oster et al. ${ }^{1}$ reported their finding of room-temperature phosphorescence in plastics containing dissolved aromatic hydrocarbons or dyes.

We wish to direct attention to work in the laboratories of the General Post Office Engineering Research Station in 1957 and onwards which resulted in the preparation of phosphorescent plastics having long decay times at room temperature. The work has been referred to elsewhere ${ }^{2}$ but has not hitherto been described in the chemical literature and may be of interest to those engaged in the examination of luminescence processes.

In a search for cheapened forms of phosphors suitable for mail-coding applications, various organic systems were examined. It was found that enhanced intensities and prolonged decay periods were given at room temperatures when certain types of activating molecule were reacted simultaneously with the major components of aminoplastic resins. Since the resins formed from cyanuric acid and formaldehyde were more easily ground to a fine powder (suitable for use in printing inks, etc.), most of the results were obtained with this system. The amino-resin phosphors have been patented ${ }^{3}$, and two such powders have been successfully used in field trials of automatic mail facing and sorting. 